\title{
Smokers' perspectives of tobacco dependency medications and medication adherence during a quit attempt in an urban NHS stop smoking service : a qualitative study
}

Carol Jane Sanders ( $\sim$ cJS342@student.bham.ac.uk)

University of Birmingham College of Medical and Dental Sciences https://orcid.org/0000-0003-11027973

\section{Antje LINDENMEYER}

University of Birmingham College of Medical and Dental Sciences John MARRIOTT

University of Birmingham College of Medical and Dental Sciences

Research article

Keywords: smoking, smoking cessation, medication adherence, qualitative research, PRIME theory

Posted Date: March 13th, 2020

DOI: https://doi.org/10.21203/rs.3.rs-17174/v1

License: (c) (1) This work is licensed under a Creative Commons Attribution 4.0 International License.

Read Full License 
1 Smokers' perspectives of tobacco dependency medications and

2 medication adherence during a quit attempt in an urban NHS

$3 \quad$ stop smoking service : a qualitative study

4

5 C.J. Sanders, A. Lindenmeyer \& J.F. Marriott

6

7 Authors

8 Carol Jane SANDERS MSc, PG Cert (HE), RN (Adult)/RSCPHN (OH)

9 PhD Student

10 Institute of Clinical Sciences

11 College of Medical and Dental Sciences

12 School of Pharmacy

13 University of Birmingham

14 Edgbaston

15 Birmingham

16 B15 2TT

17 UK

18 Telephone: +4407592606065

19 Email: CJS342@student.bham.ac.uk

20

21 Antje LINDENMEYER MA PhD

22 Social Studies in Medicine

23 Institute of Applied Health Research

24 Murray Learning Centre

25 University of Birmingham

26 Edgbaston

27 Birmingham

28 B15 2TT 
Telephone: +44 (0)121 4145390

Email: a.lindenmeyer@bham.ac.uk

32

John MARRIOTT MR Pharms FHEA

Professor of Clinical Pharmacy

Institute of Clinical Sciences

College of Medical and Dental Sciences

School of Pharmacy

University of Birmingham

Edgbaston

Birmingham

B15 2TT

UK

Telephone: +44 (0)121414 4094

Email: j.f.marriott@bham.ac.uk

45

Correspondence Author: Mrs C.J. Sanders: email CJS342@student.bham.ac.uk

47

\section{ABSTRACT}

Background: Smoking and poor medication adherence are both associated with increased morbidity, mortality and immense costs for the healthcare system. Numerous studies have investigated the barriers and facilitators of medication adherence among patients with chronic disease. However, the factors influencing adherence to medication targeted to reduce smoking remain unexplored. In order to achieve further reduction of smoking prevalence, improvement of medication adherence is essential. Thus, this study aims to uncover and understand adult smokers' attitudes towards adherence to medication to reduce tobacco dependence. 
Methods: A purposive approach was used to recruit smokers aged over 18 and motivated to quit using pharmacotherapy. In-depth semi-structured interviews were conducted with a diverse sample of eleven smokers who were engaged with a NHS Stop Smoking Program. Data were thematically analysed using PRIME theory.

Results: Smokers were active decision makers regarding their tobacco dependence medications. Adherence was influenced by smokers' evaluations, plans, access to support and experiential learning, highlighting the need for tailored adherence support. Conflicting views about medication were held by all participants. Side effects, fear of dependency and inability to stop cravings influenced initiation and adherence. Electronic cigarettes were viewed negatively. Adherence was influenced by their unique psycho-social contexts including deeprooted personal rejection and, fear of medicines. These attitudes influenced smokers' beliefs, decisions about quitting and the role and use of medications in the quitting process.

Conclusions: Tobacco dependency is a unique treatment context with specific adherence issues. Lay perspectives of medications to support quit attempts differ from the medical viewpoint. Adherence to tobacco dependency regimens is a challenging goal, inhibited by many complex factors. There is a need for smoking cessation interventions to respond to individual medication beliefs and concerns to help to build a smoker's confidence that an individual can take their tobacco dependency mediation as prescribed and maximise benefits.

Keywords smoking, smoking cessation; medication adherence; qualitative research, PRIME theory.

\section{BACKGROUND}

Worldwide, smoking remains the most important preventable cause of premature death and disability (1). Smoking contributes to health inequalities, causes $16 \%$ of all UK deaths (2) and costs the National Health Service (NHS) circa £2bn annually (3). Tobacco dependency medications (TDMs) effectively aid initial abstinence and decrease lapse risk (4); however, 
globally, most smoking cessation (SC) attempts are unassisted. NHS stop smoking services (SSSs) provide evidence based and cost-effective support to quit attempts $(5,6)$. Evidence indicates that, although the majority of current smokers are motivated to quit, the number of people using NHS SSSs to help them quit continues to decline (7). This may be because in recent years, e-cigarettes have become prevalent, generating uncertainty within clinical SC practice and amongst smokers. There is a lack of international consensus regarding the role of using e-cigarettes as a tool for SC. Public Health England (PHE) and NHS Health Scotland advocate e-cigarettes in contrast to other public health bodies such as the World Health Organisation. A recent UK trial found that, smokers who used e-cigarettes when combined with behavioural support to quit smoking were twice as likely to succeed as smokers who used other nicotine replacement products (8). In contrast, a qualitative study found that both smokers seeking to quit and SC advisors had ambivalent attitudes towards e-cigarettes with regards to efficacy, health risks and incompatibility to SC goals (9). Overall, long-term quit success through NHS SSSs remains low (10) partly explained by poor TDM adherence.

Medication adherence, defined as the extent to which patients take medication as prescribed by their healthcare professionals (HCPs) is an important aspect of treatment efficacy, healthcare costs and patient safety (11). Internationally, medication non-adherence is recognised as a major public health issue with serious consequences (12). It is estimated that $£ 300 \mathrm{~m}$ of NHS prescribed medicines are wasted each year (13). Factors that influence adherence to TDMs remain unclear; for example, we know little about the ways in which smokers perceptions and experiences of TDMs influence adherence. A recent meta-ethnography (14) found that smokers are active decision makers regarding using TDMs. For HCPs to work in partnership with smokers, knowledge of smokers' poor TDM adherence during quit attempts is required. Research on lay perspectives regarding adherence to TDMs is sparse and there remains a paucity of literature to inform the design of effective TDM adherence promoting interventions. 
Adhering to prescribed medication regimens is a complex behaviour requiring the ability to access prescribed medication and the cognitive capacity and motivation to take it. Nonadherence may occur at initiation (the medication is not started), implementation (delay, omitting or taking extra doses during treatment) or persistence (premature discontinuation) phases of medication use (15). There are many reported barriers and facilitators of medication adherence (16); in particular, patients' treatment-related beliefs are important for their adherence behaviours as medication-related concerns and necessity-beliefs predict adherence (17). The perspective of patients, carers and HCPs often differs in terms of the determinants of medication adherence owing to priorities and knowledge of the situation. Estimates of the rate of adherence to TDMs ranges widely between studies and countries (12). Additionally, tobacco dependency (TD) is a unique treatment context with specific adherence issues. TDM adherence is shaped by several complex factors: dependence on tobacco, preference not to use medications, fear of side effects, depression, gender, weight gain, cravings, withdrawal symptoms, low motivation to quit smoking, poor social support and fear of dependency on TDMs (16). In contrast, motivation, access to free TDMs and SC support have been found to increase adherence to TDMs (18).

There is a strong relationship between cigarette smoking and social disadvantage (19). Smokers from deprived areas have low awareness of services available to help them, and hold misconceptions about their availability and effectiveness (20). Previous studies have found several factors that partly explain TDM underuse such as side effects, cost and access, satisfaction, lack of awareness or knowledge, prior experience of using TDMs, perceived poor efficacy or concerns about safety and dependency (16). Adherence to medication may be determined by other factors such as socioeconomic resources and individuals' beliefs trust or expectations. Poor medication adherence is considered a potential contributor to disparities in health outcomes (21). 
Theories of behaviour change stress the central importance of an individual's motivation to quit an addiction. Contemporary behaviour change frameworks such as the Theoretical Domains Framework (22) and Behaviour Change Technique (BCT) taxonomy (23) have been used across a range of health behaviours, and they are increasing applied within medication adherence research. The seminal work of Horne and colleagues (17), the Necessity-Concerns Framework, suggests that adherence is influenced by implicit judgements of personal need for the treatment (necessity beliefs) and concerns about the potential adverse consequences of taking it. The PRIME (Plans, Responses, Impulses, Motives, Evaluations) theory of motivation (24) has been applied to smokers in attempting to explain how quit attempts are made. PRIME theory states that the decision to quit smoking is based on a smoker's evaluative beliefs (positive or negative) about smoking, which influence motivation to quit or not. This involves internal tensions (impulses and urges to smoke) and external triggers (e.g. cues in the environment) to determine subsequent behaviour.

A comprehensive review of interventions to increase medication adherence and improve related outcomes using a variety of approaches found their effects were inconsistent; only a few clinical trials improved both adherence and clinical outcomes (25). The most effective medication adherence interventions adopted comprehensive approaches involving a range of strategies, were high-intensity, and tailored to individual patients (26). HCPs (i.e., physicians, nurses, pharmacists) played a key role in supporting, promoting and monitoring medicines adherence in chronic conditions. Patient-centred approaches to improve adherence are considered promising (27); however their effectiveness remains unclear. In the absence of a single definitive intervention to address non-adherence, current NICE Guidelines combine trial evidence of interventions and explanatory studies of non-adherence (28). The dearth of evidence underpinned by health psychology theory is of concern given its importance for informing intervention design and implementation. In order to understand the potential of 
interventions supporting TDM adherence, it is important to explore views and attitudes towards smoking, quitting, and TDMs. Using the conceptual framework of PRIME theory, this article reports on a UK-based qualitative study exploring in-depth, smokers' beliefs, experiences and attitudes towards TDMs to help explain poor TDM adherence. The study findings are of value to clinicians, nurses, policymakers and researchers as it seeks to inform future design of SC interventions that enhance TD treatment engagement, adherence, and long term quit outcomes.

\section{METHODS}

The authors conducted a qualitative study based on in-depth interviews with smokers attending an urban NHS Stop Smoking Service in Birmingham, UK between November 2014 and April 2015. As our aim was to uncover and understand adult smokers' TDM adherence from their own perspective, we used this methodological approach, which allows a flexible exploration of participants' experiences and beliefs $(29,30)$.

\section{Recruitment and eligibility of study participants}

A purposive approach was used to recruit a sample of smokers aged over 18 currently engaged in quit attempts using TDMs. Participants were recruited from a single residential area in Birmingham and received no reimbursement for taking part. Advertising posters inviting smokers to discuss their opinions about TDMs were placed in the SSS. The SSS SC Advisers asked for interested people to volunteer to take part. Participants were enrolled into the study after they had selected their TDMs. In line with current clinical SC support this was ahead of their quit date in Week One of their behavioural support group (31). The present study set out to obtain interviews from each participant four weeks after their quit date. Recruitment aimed to obtain enough data to sufficiently describe the phenomenon of interest and address the research question (29). 
182 Data was collected between November 2014 and April 2015. An initial meeting was set up with each participant, prior to arranging a date and time for the face-to-face interviews in which participants consented, in writing, to the study after full explanation of what was involved. All the interviews were conducted by CJS. A short survey was completed by participants before the start of the interviews to gain insight into their demographic characteristics, TDM use, smoking history and TD (assessed using the Fagerström Test for Cigarette Dependence (FTCD) (32)).

An interview guide (Additional File 1: In-depth interview guide) was developed to explore how the views, beliefs and experiences of adult smokers shaped their adherence behaviours. Interviews lasted up to $90 \mathrm{~min}$ in a private room within an NHS SSS and were audio recorded. The main topics were experiences, views, meanings and beliefs held about TDMs and ecigarettes, barriers to adherence and suggestions for reducing these barriers. Questions were guided by concepts from PRIME Theory, for example asking for details of plans to quit or return to smoking and evaluative beliefs about smoking and TDMs.

Additional File 1: In-depth interview guide

\begin{tabular}{|l|l|l|}
\hline Category & Questions & Probes \\
\hline $\begin{array}{l}\text { Knowledge } \\
\text { and } \\
\text { experiences } \\
\text { of } \\
\text { medication } \\
\text { to aid } \\
\text { smoking } \\
\text { cessation }\end{array}$ & $\begin{array}{l}\text { What kind of support do you need and/or } \\
\text { prefer in dealing with your experiences and } \\
\text { symptoms of quitting smoking? } \\
\text { What is your view on the medication } \\
\text { methods for people trying to quit? }\end{array}$ & $\begin{array}{l}\text { Do you think it is necessary to use TDMs to } \\
\text { help you stop smoking? Do you think it is } \\
\text { safe? } \\
\text { to aid a quit attempt, what has } \\
\text { supported past quit attempts, } \\
\text { evaluations of the causes of relapse to } \\
\text { smoking) }\end{array}$ \\
$\begin{array}{l}\text { changed your views of them? } \\
\text { Do you adhere (stick to) to other medication } \\
\text { differently to TDMs? }\end{array}$ & $\begin{array}{l}\text { (Probe: emotional issues, impulses } \\
\text { that undermine adherence, do you } \\
\text { think it is effective?) } \\
\text { (Probe: how has knowledge and } \\
\text { understanding changed over the quit } \\
\text { journey?) }\end{array}$ \\
\hline $\begin{array}{l}\text { Medication } \\
\text { use and } \\
\text { adherence }\end{array}$ & $\begin{array}{l}\text { Tell me about all the medicines that you are } \\
\text { taking currently. }\end{array}$ & $\begin{array}{l}\text { (Probe: names, doses, and timings, } \\
\text { motives for choosing to use a } \\
\text { medication to quit) }\end{array}$ \\
\hline
\end{tabular}




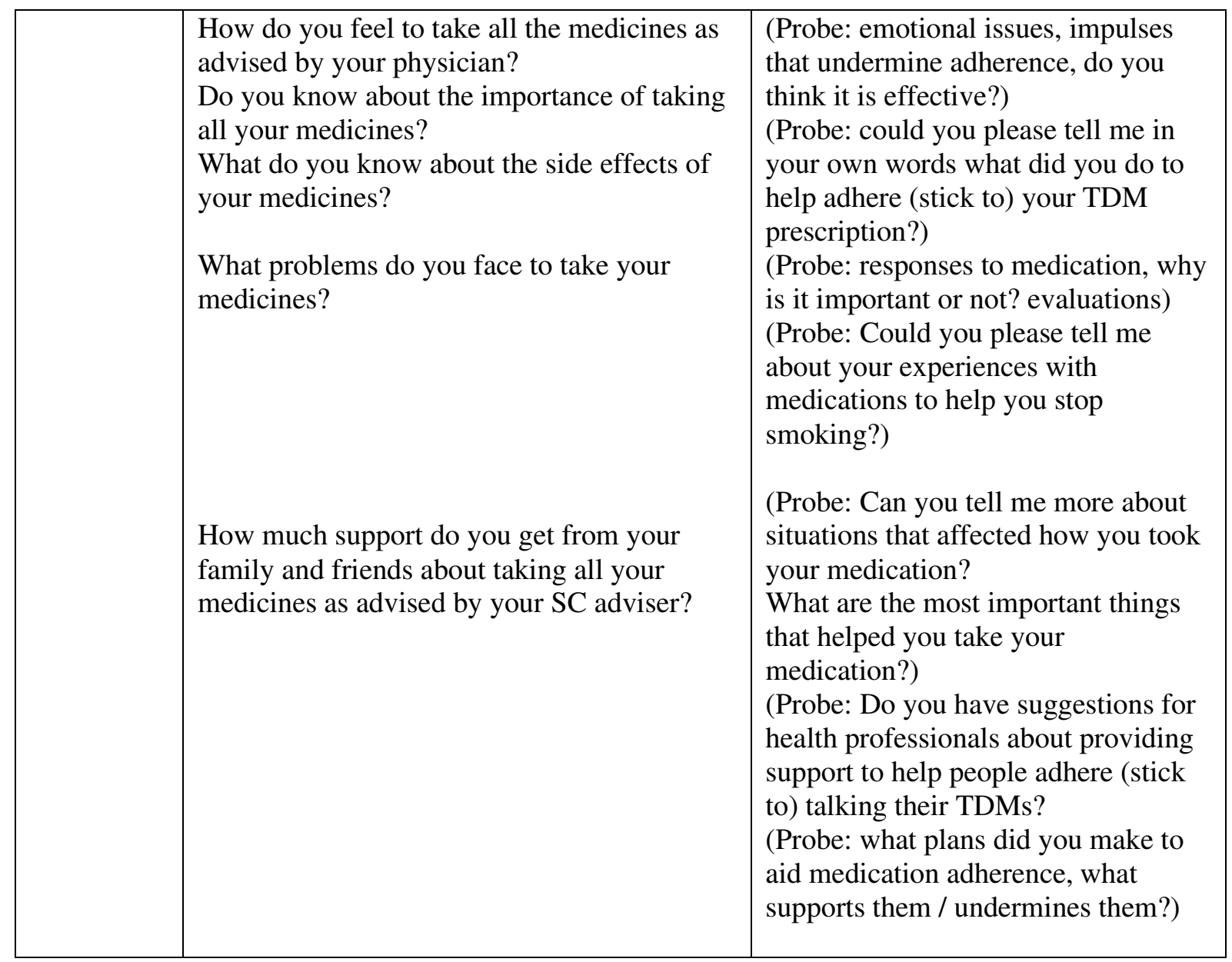

Smokers were provided with a Participant Information Sheet during their SSS clinic visit outlining details of the study and the opportunity to contact the researcher if they had any enquiries before confirming their involvement. All participants were assured of confidentiality, the anonymous processing of the data and that SSS staff did not have access to the data. The researcher (CJS) had no access to SC patient records. Ethics committee approval was obtained Birmingham.

Analysis 
of codes in 'higher order' analytic themes to produce an interpretation. The analysis was both inductive, from the accounts (experiences and views) of participants, and deductive, through reflected in the study objectives and the topics chosen for the interviews). The first draft of the analysis was generated by the first author and reviewed/reworked by all authors until interpretation was agreed. This process identified meaningful extracts of text used to identify, label and refine the themes which best explained the data. To assure methodological rigour, authors undertook an "audit trail" outlining decisions made throughout the process of data collection and analysis (34). Authors documented their discussions on coding and analysis of data as a method of checking plausibility of emerging themes, the paths taken to arrive at those themes and data interpretations $(29,33)$. In addition, the transcribed data allowed all authors to revisit the text to check emerging themes and ensure that they remained true to participants' accounts. Details of demographics, smoking characteristics, TDM and adherence history are described in (Table 1: characteristics of study participants) allowing readers to contextualise participants' responses. COREQ guidelines were followed in reporting the methods (35).

Table 1. Characteristics of study participants

\begin{tabular}{|l|c|}
\hline Characteristic & $\mathbf{n = 1 1}$ participants \\
\hline Mean age, years (SD) (range) & $47(11.27)(31-65)$ \\
\hline Gender, percent (number of participants) & $55 \%(\mathrm{n}=6)$ \\
Female & $45 \%(\mathrm{n}=5)$ \\
Male & $100 \%(\mathrm{n}=11)$ \\
\hline Ethnicity, percent (number of participants) & $15(13-17)$ \\
White, British & \\
\hline Age of smoking initiation, years (range) & $82 \%(9)$ \\
\hline Fagerström score, percent (number of participants) & $18 \%(2)$ \\
7 to 10 points = highly dependent & $0 \%(0)$ \\
4 to 6 points = moderately dependent & $0 \%(0)$ \\
less than 4 points = minimally dependent & $18 \%(2)$ \\
\hline Number of cigarettes smoked per day, percent (number of participants) & $18 \%(2)$ \\
10 or fewer & $64 \%(7)$ \\
11-20 & \\
21-30 & \\
31 or more & \\
\hline
\end{tabular}




\begin{tabular}{|l|c|}
\hline Number of cigarettes smoked per day (cpd), Mean (range) & $32 \mathrm{cpd}(20-60)$ \\
\hline Time to first cigarette of the day, percent (number of participants) & $36 \%(4)$ \\
$<5 \mathrm{~min}$ & $45 \%(5)$ \\
$5-30 \mathrm{~min}$ & $18 \%(2)$ \\
$31-60 \mathrm{~min}$ & $0 \%(0)$ \\
$>60 \mathrm{~min}$ & \\
\hline Ever used nicotine replacement therapy in attempt to quit percent & $8(73 \%)$ \\
(number of participants) & $3(27 \%)$ \\
Yes & \\
No & \\
\hline Ever used pharmacotherapy (Varenicline (Champix) or Bupropion & $10(91 \%)$ \\
(Zyban) in attempt to quit percent (number of participants) & $1(9 \%)$ \\
Yes & $40(4)$ \\
No & \\
\hline Number of previous quit attempts, total for cohort, Mean & $55 \%(6)$ \\
\hline Adherence behaviour group, percent (number of participants) & $27 \%(3)$ \\
Group A: fully adherent & $18 \%(2)$ \\
Group B: partially adherent & \\
Group C: nonadherent & \\
\hline SC medication used to aid their current quit attempt, percent (number of & \\
participants) & $18 \%(2)$ \\
NRT (any form of NRT such as gum, patch, inhaler, tablet/lozenge, nasal & \\
spray) & $73 \%(8)$ \\
Champix & $0 \%(0)$ \\
Zyban & $9 \%(1)$ \\
No SC medication & \\
\hline
\end{tabular}

\section{RESULTS}

Eleven adult smokers registered with an urban NHS SSS participated in the study. The

participants were all long-term, highly nicotine dependent smokers who had failed several previous SC attempts and had a varied adherence history with TDMs. All participants stated that they were motivated to quit and had chosen to use TDMs to support their quit attempt. The process of defining and labelling the themes was guided by the study objectives, Prime Theory and new concepts developed inductively from the data. Key categories identified in our data were mapped against motivational reasons that may have influenced non-adherence. We were encouraged by the consistency between our categories and the concepts of Prime Theory i.e. plans, responses, impulses and inhibitory forces (urges), motives (feelings of want or need) evaluations (evaluative beliefs) and the findings of previous research. 
Our central analytical focus, however, were the original, previously unreported themes in our analysis. When grouped, these indicated four new processes that could help explain TDMs adherence behaviours:

1. Evaluating TDMs;

2. Planning to use TDMs;

3. Available support;

4. Experiential learning.

\section{Evaluating TDMs}

PRIME theory states that "we arrive at evaluations through a process of generalisation and deduction". Participants reminisced about their smoking in positive and negative terms which influenced their acceptance of TDMs. Cognitions about SC were deeply embedded in participants' experiences which evoked their emotions, shaping their ideas about TDMs.

$$
\begin{aligned}
& \text { "I've got the NRT, why do I need it? I ask myself, why?” (P4) } \\
& \text { “...I need to have something to help me stop...” (P8) }
\end{aligned}
$$

TDM experiences led participants to believe that they were not a complete solution; mostly owing to TDMs' inability to stop NWS. Participants' beliefs had both positive and negative dimensions which affected motivation to use TDMs. Participants' evaluations of TDMs were expressed as conflicting emotions and beliefs while reflections on past quit attempts motivated participants to use TDMs. Reflections on past relapses also motivated participants to use TDMs. Beliefs about TDM evolved increasing confidence in TDM efficacy. it too hard...' (P14) 
Most participants articulated strong fears of TDM use (e.g. side effects, dependency). They assessed the risks and benefits of TDMs within their psychosocial context: these TDM evaluations were an important facet of TDM adherence:

“...it was so hard, with the mood swings and stuff like that, and I felt really down when I was on the Champix (P2)

“...perhaps if I had more motivation, I may have had more tolerance to the side-effects, but when I look back on it I just think it was a horrible experience". (P6)

This indicates that HCPs should tailor their TDM advice in line with an individual's beliefs to increase TDM adherence.

\section{Planning to use TDMs}

Plans, according to PRIME theory "allow for behaviours to be deferred according to priorities or anticipated opportunity or threat (36). The process of planning seemed more important to some participants than the plans that emerged; it provided smokers with "thinking time" about past failed quit attempts. They were frequently challenged by the pressures of addiction to cigarettes which undermined adherence.

'This is really hard. I'm struggling. I think on Saturday I'm going to go out and have a few drinks. I won't wear a patch, and I'll smoke.' (P7)

"I can't do it without them. So, I guess to me they're essential. I feel that I couldn't cope without the patches, or the lozenges now, but having said that, I'm quite dependent on them. (PI)

TDM use reflected participants' (i) realisation they needed TDMs in order to implement their desired behaviour change in the face of conflicting wants, needs and urges; (ii) understanding 
that TDMs provided a way forward that minimised NWSs and (iii) required a shift in deep identity - from smoker to non-smoker.

“... the mistakes that I've made, I've rectified them this time around, whereas in the past I used to leave it too long in the morning before having any NRT..." (P5)

In contrast, participants' who were not fully committed to SC made choices in the way they used TDMs that enabled them to smoke.

"I'd smoke through the Champix and make myselffeel really ill, and then I'd think, the next day, 'Oh, well, I gave in yesterday. I'm not going to take them [sic Champix] today,' and I'd deliberately not take them $(P 2)$

Partial adherence, described by some participants, occurred in response to impulses or urges to smoke. Participants then made independent decisions about increasing or decreasing their TDM doses. Planning activities emerged as useful to achieving TDM adherence. PRIME theory identifies that acting in pursuit of what we most want and need is a central feature of addictive behaviour (37). Participants deviated from their plans to use TDMs in response to various stimuli, commonly the urge to smoke, habit, psychosocial factors e.g. significant others, negative emotions:

“I'd deliberately not take Champix for a couple of days because I wanted to smoke..."(P11)

Participant's narratives revealed the importance of coping, reassurance and predictability that came with having a cigarette. These needs were not fully replaced by TDMs. They suggested that the prerequisites of an effective TDM was that it replaced the cigarette niche in their life. Conversely, as none of the participants wanted to be viewed as an addict, they believed TDMs should not have characteristics of a cigarette or contain "the enemy" nicotine. All participants were aware of e-cigarettes and a predominant narrative was that they wanted certainty about their safety and role within SC. Two participants felt that e-cigarettes mimicked smoking a real 
cigarette and believed that that the use of them was either ineffective or an indication that a person was not ready to quit smoking.

“...these e-cigarettes, and I've been told that because there's been no research into them no one really knows how effective they are or what they do to you... what damage they can do in the long run...I personally don't see them as a replacement, although others obviously do because they are stopping with them...” (P2)

"Before I started out on the patches, I wanted to do the e-cigs, because I'd heard so many good things about them. But it was the fact that they weren't licensed that put me off..." (P3)

"It makes no difference whether it's an e-cigarette or a Benson and Hedges, you're still doing the same habit..." (P6)

Participants did not recognise that their adherence was "poor" in clinical terms and it was not given as the reason for the difficulties of SC. Participants conveyed how they had actively selected different TDMs over the course of their journey to stop smoking. They expressed a limited confidence in TDMs with anger and/or denial about their smoking addiction. Some said they quickly stopped TDMs in response to side effects and worried about replacing their addiction to cigarettes with an addiction to medication. These converging cognitions destabilised TDM adherence. Participants displayed differing opinions regarding TDMs; some believed that they were essential to their SC plans, whereas others did not. Most participants held negative views towards TDMs stating that they were concerned about the side effects and were not confident about TDM efficacy. Some participants also held expectations that TDMs were a "quick fix" for their smoking addiction, believing it was their last hope for becoming a non-smoker. Others liked the relief and confidence TDMs provided. Participants' past experiences of TDMs helped to build a schema that was drawn upon to form plans for their subsequent quit attempts. This meant that plans to use TDMs were affected by what happened around them which determined their TDM adherence behaviours. 


\section{Available support}

All participants' TDM adherence behaviours were influenced by various social interactions. TDM adherence involved positive (supportive) behaviours by their social circle, such as shared decisions about which TDM to use, and reinforcement such as agreement on how to use TDMs. "We both said that if we were going to do it, it would be with Champix. I think we both see it (Champix) the same way. It's just a tablet. Pop it, finish for the day, sort of thing. Get on with it”. (P4)

In contrast critical (negative) behaviours, such as challenging the participant's smoking, shared complaints/concerns about TDMs or agreed decisions to smoke, led to poor adherence. Family context and cohabitants' smoking status (social support) were influential to participants' SC attempts and decisions regarding TDMs access and adherence behaviour.

"smoking has been my thing and a lot of people round me that have said, oh you know, especially my partner, 'Oh, it is easy to give up, you just make a choice'... to them that might be possible but I find it incredibly difficult to give up without some support. (P7)

Some participants portrayed chaotic lives, carelessness with TDM use and intentional nonadherence. Participants' reported stress, conflict and anxiety because of trying to rationalise smoking with family members' concerns regarding the dangers and harms associated with smoking. Participants' addiction to smoking had many significant and enduring impacts on family dynamics and functioning. A greater role for family support might be considered regarding supporting use and adherence with TDM regimens. PRIME theory states that environmental cues can influence the decision to smoke by triggering impulses. SC support accessed by participants influenced TDM adherence behaviours. Most participants were inspired to make a quit attempt by the Stoptober campaign (38) which provided a supportive social context to attempt to quit. Participants found accessing the SSS helpful in providing 
support to their TDM use. The SSS role was a place to access TDMs but also met participants' social and emotional support.

"When she'd explained what patches did... what Champix did, because she was telling me that you just take a tablet and then it makes you stop smoking, I thought, 'Great.' So that choice was made by the information that she'd given me at the clinic." (P2)

Narratives contained several interactions with HCPs that were very positive and a main source of advice regarding the selection and management of TDM, side effects and regimen modification. However, not all interactions with HCPs were positive. One participant was frustrated that although he did not want to use NRT, the SC adviser issued it regardless:

"I did say to the SC Adviser that I didn't want any medication and she said, 'But when the nicotine receptors in your brain start crawling round for nicotine, you're going to find it very, very hard'. That's the only thing I disagree with her about. I think she could probably find out more about the person, before shoving the medication at them" (P6)

Another participant recalled a negative experience regarding access to his Champix prescription, the HCP was perceived as unhelpful and unfortunately the participant returned to smoking. Most participants preferred having an active role in TDM decision making which had important effects on adherence:

"I tried again, about three years ago, on Champix... Every time I'd go the prescription wasn't ready or he couldn't see me or he was really busy, so I'd go two, three days without having anything at all. So, I just automatically started smoking again...I think if he was more reliable, I don't think I would be smoking now...” (P4)

\section{Experiential learning}


Participants described in detail their smoking addiction and struggles to stop; this formed a highly negative context for achieving adherence to TDMs. They all had unique histories and relationships with TDMs which shaped their adherence behaviours. Learning from experiences with TDMs led to an increased perception of their role with reducing NWSs; this strongly influenced participants' TDM adherence. When a TDM stopped urges to smoke and NWSs, participants were more likely to persist with TDMs.

“...what I've learnt from the two failures before, is that you must complete the course...” (P7) “what I did learn about the Champix, I found that there was a learning curve...don't ever think that it is a magic pill; it's not a magic pill, but what it does is, it's a blocker." (P7)

"I'm starting to learn more how they (NRT patches) affect me and how I need to do it, to change it, do you know what I mean..." (P4)

While participants acknowledged TDM benefits, they held strong concerns about dependency and side effects. They described how TDMs added to their problems, particularly noting the experience of intense emotions or unpleasant bodily effects caused by TDMs. The most common reasons given for not taking TDMs were side effects, forgetfulness, deciding to omit a dose, other priorities and emotional reasons which caused changes in physical or mental status. Previous experiences with TDMs that generated unpleasant side effects delayed future initiation of TDMs; significantly some participants associated TDMs with a failed quit attempt that made them feel horrible.

\section{"I felt really down when I was on the Champix (P2)}

"I think for me it didn't help to know that it was blocking the nicotine, because that just made me think, 'Oh, I'm going cold turkey." (P9)

"I think with the Champix it's blocking me getting nicotine, but with these patches, they're giving me nicotine" (P11) 
Asking participants to talk about how they found out about TDMs provided new insights into why TDM adherence is often poor. There exists a disjoint between smokers and HCPs about TDM efficacy; participants' adherence was strongly influenced by their experiential learning rather than advice they received from HCPs. The experiential learning of TDMs formed participants' judgements of TDM efficacy; adherence behaviours were susceptible to participants' experiences.

"When you use Champix, you get up in the morning and you haven't got the urge for a cigarette...I can't describe it, it's just weird. They are the best thing ever." (P6)

Participants had limited faith (confidence) in TDMs, exhibited anger or denial about their addiction and quickly rejected TDMs in response to side effects experienced.

"Champix, I found them potent, and I didn't like putting them into my body because of what they did to me, in terms of not feeling well, and the mood and the stomach problems. (P5)

All participants expressed a strong view of not wanting to replace their addiction to cigarettes with an addiction to TDMs. These converging cognitions were key deterrents to TDM adherence. Participants were unable to express their behaviours in the same terms as HCPs; adherence was not a concept/term they knew. In stressing the negative aspects and addictive potential of TDMs, participants expressed views about TDMs that opposed medical knowledge of TDM benefits.

Incorporating the conceptual framework of PRIME theory with the four themes found in the present study, we conceived a model of smokers' TDM adherence (Figure 1). The model depicts the dynamic cognitions and complex processes that, within the context of TD, affect adherence; this model may be helpful to SC policy and clinical practice guideline formulation. This model seeks to help optimise adherence with TDMs and contribute to improving the desired outcomes of SC and avoidance of pharmaceutical waste. Improving the ability to properly assess the risk 


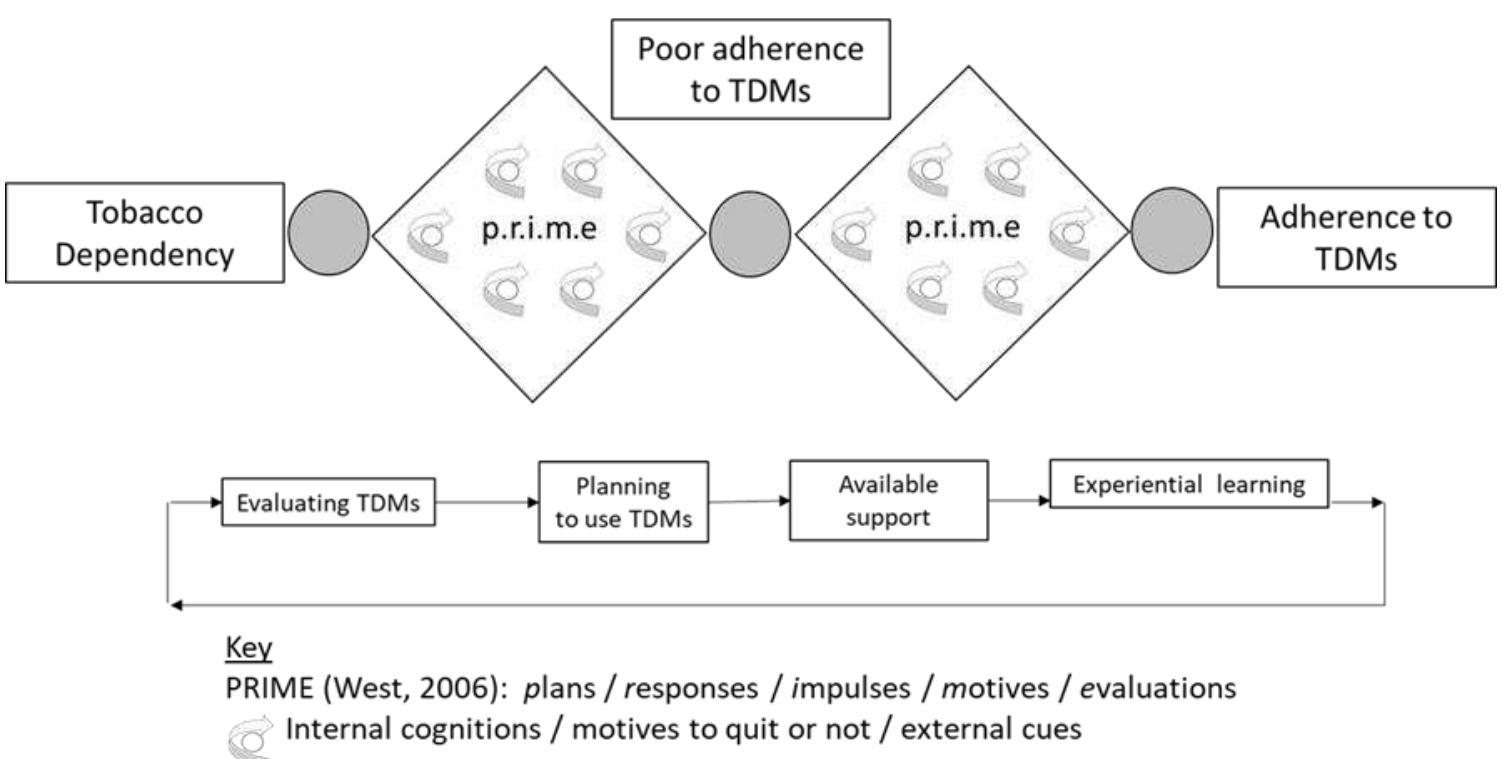

\section{DISCUSSION}

This qualitative study aimed to understand adult smokers' poor TDM adherence during quit attempts via a qualitative approach using the conceptual framework of PRIME theory. In this sample of smokers seeking to quit via an NHS SSS, the processes of evaluating TDMs, planning to use them, available support and experimental learning affected TDM adherence behaviours. TDM adherence has been investigated before (16); the exploration of how smokers' views, beliefs and experiences of TDMs

442 shaped their adherence behaviours during their quit attempts is particularly unique to this study. As with most smokers $(14,39)$, unassisted quitting was perceived as the optimal way to stop smoking. A history of failed quit attempts caused participants to feel inadequate and frustrated that their willpower alone had not enabled them to quit. Motivation, willpower and commitment have been found to be important to smokers who quit without TDMs (Smith, Chapman, \& Dunlop, 2015); all participants understood that those factors affected their SC success. Utilising beliefs about the importance of willpower in SC clinical interactions may be important to improving TDM adherence. 
PRIME Theory emphasises plans to develop strategies that assist smokers with quitting smoking. Planning emerged as an intrinsic part of achieving TDM adherence. We found many complex influences leading to poor TDM adherence. According to PRIME Theory, an individual's evaluations inform their motives and intentions to plan a certain course of behaviour. Participants' evaluations, critically of their smoking addiction and past failed quit attempts, eventually led to learning about the role of TDMs and to acceptance that medication was important to their SC solution. Participants cited the inability of TDMs to stop cravings (impulses and urges) for a cigarette as the reason for stopping TDM use. Over time, participants transitioned from unfamiliarity and rejection of TDMs towards critically accepting the role of TDMs in undoing their addiction. The main driver of this transition was experiential learning from past failed quit attempts. This led to acceptance of the need to prevent/respond to impulses to smoke with TDMs. Participants were distressed about their smoking addiction and this transferred into fear of becoming dependant on TDMs. Participants' rejection of TDMs, influenced by strong beliefs in unassisted quitting, has been found in other studies $(40,41)$. This belief augments concerns about TDMs and is a factor in poor TDM adherence.

Our finding that participants commonly did not use TDMs in accordance with clinical recommendations is consistent with evidence from other studies $(12,42,43)$. Poor TDM adherence increased participants' experience of NWS which further weakened perceptions of TDM efficacy. Deliberate non-adherence mostly occurred owing to relapse to smoking; the lived world of nicotine addiction undermines TDMs adherence. Poor TDM adherence has been explained in other studies as being due to: a high level of dependency (44), low mood (45), concerns about side effects and future adverse effects (16) and nicotine withdrawal symptoms $(46,47)$. We found that participants' attitudes towards TDM options were formed by several diverse, converging, dynamic issues. Participants expressed several concerns about electronic cigarettes (ECs) i.e. that they mimicked smoking, undefined safety, potential for nicotine dependence, and sought evidence of safety from HCPs. There is evidence that ECs help smokers to stop smoking in the long term compared with placebo ECs (48). In our study, the perceived role of ECs as a quit aid was uncertain amongst participants who had concerns about safety and nicotine dependence. This finding is contrary to current academic thought that ECs may have an important role in harm reduction (49). Some 
participants, as with smokers in general (50), viewed ECs tentatively as being less harmful than smoking

477 but were unsure of their efficacy as aids for SC.

478 Arguably, the current treatment approach has an overemphasis on the medical model, which underestimates the impact of complex TDM taking cognitions and behaviours that occur. Most participants depicted their smoking behaviour as a "response" (a concept of PRIME Theory), to external triggers (social influences) and internal cues (self-image). Participants' narratives revealed that their TDM adherence behaviours were underpinned by responses to complex psychosocial factors and addiction which caused impulsive and conflicted decision making. The study found considerable reluctance to take TDMs and a preference to take as little as possible for a short duration. This may partly explain why adherence to TDMs has been found to be very low in the long term $(<40 \%)(12)$.

We found that each participant's experience of TDMs was deeply personal and their judgements were influenced by their individual context and personality. Improved TDM adherence may be facilitated by a greater emphasis on collaborative approaches that recognises the expertise of both the person attempting to quit smoking and HCPs. Participants did not passively accept what HCPs told them about the need for or how to use TDMs. Understanding of smokers' perceptions of TDMs is an important element of establishing an effective therapeutic relationship. HCPs need to identify where people with TD are in relation to rejection and initiation in TDM decision-making in order to work with them to achieve TDM adherence. It is also important TDM adherence is viewed within a broader context of a holistic framework rather than from a narrower medical discourse. Future efforts to improve adherence to TDMs may need to take the processes outlined in this study into consideration.

\section{LIMITATIONS}

The qualitative design of this study allowed us to extend the existing literature on TDM adherence to provide a more in-depth discussion of the complex reasons for why adherence to TDMs is poor. An important strength of this study was the sample's in-depth experiences of SC and TDMs. The qualitative nature of our study limits the generalisability of our findings to a broader population of individuals with TD. Furthermore, the study was conducted in a single NHS SSS situated in a deprived area. Participants 
we recruited had several prior attempts at SC and experience with TDMs and may not reflect the experiences of smokers seeking to quit for the first time. Therefore, results may be not generalisable to smokers in other settings. ECs have emerged as a controversial SC method, but we did not explore directly its role in increasing adherence to TDMs.

\section{CONCLUSION}

Adherence to TDM regimens is a challenging goal, potentially sabotaged by many complex factors. Smokers TDM literacy and contextual influences are important to TDM adherence. Poor adherence may be difficult to comprehend, though this study does provide some explanation using the conceptual framework of PRIME Theory. ECs were viewed negatively by participants who voiced the need for further authoritative safety information. Adherence to TDMs was influenced by smokers' evaluations, plans, access to support and experiential learning highlighting the need for individualised TDM adherence support. The findings of this study are important for future TD clinical practice guideline development if HCPs are to contribute to the further reduction of smoking prevalence. As this was qualitative research, no inferences about the prevalence of these beliefs, experience and attitudes towards TDMs in the larger population of smokers can be drawn. However, these findings provide valuable insights into complex processes that influence TDM adherence. Interventions to improve adherence to TDMs need to acknowledge and incorporate smokers' concerns and perspectives.

\section{ABBREVIATIONS}

HCPs: healthcare professionals

NHS: National Health Service

PHE: Public Health England

SC: smoking cessation

SSSs: NHS stop smoking services

TD: Tobacco dependency 
TDMs: Tobacco dependency medications

530

531

532

533

534

535

536

537

538

539

540

541

542

543

544

545

546

547

548

549

550

UK: United Kingdom

\section{DECLARATIONS}

Ethics approval and consent to participate

Ethics committee approval was obtained from both NHS Health Research Authority (REC 13/SW/0173) and the University of Birmingham.

Consent for publication

Not applicable.

Availability of data and materials

Data sharing is not applicable to this article as no datasets were generated or analysed during the current study.

\section{Competing interests}

The authors declare that that they have no competing interests.

None to declare.

Funding

This research received no specific grant from any funding agency in the public, commercial, or not-for-profit sectors.

Authors' contributions

All authors have agreed on the final version and meet the following criteria (recommended by the ICMJE (http://www.icmje.org/recommendations/browse/roles-and-

responsibilities/defining-the-role-of-authors-and-contributors.html)):

\begin{tabular}{|l|l|}
\hline Criteria & Author Initials \\
\hline $\begin{array}{l}\text { Made substantial contributions to conception and design, or } \\
\text { acquisition of data, or analysis and interpretation of data; }\end{array}$ & CJS, AL, JM \\
\hline $\begin{array}{l}\text { Involved in drafting the manuscript or revising it critically } \\
\text { for important intellectual content; }\end{array}$ & CJS, AL, JM \\
\hline $\begin{array}{l}\text { Given final approval of the version to be published. Each } \\
\text { author should have participated sufficiently in the work to }\end{array}$ & CJS, AL, JM \\
\hline
\end{tabular}


take public responsibility for appropriate portions of the content;

Agreed to be accountable for all aspects of the work in ensuring that questions related to the accuracy or integrity of any part of the work are appropriately investigated and resolved.

\section{Acknowledgements}

We thank our colleagues at the Stop Smoking Service Adults \& Community Division Birmingham Community Healthcare NHS Trust e.g. for support with securing ethics approval, setting up the study and to Anne Fitchett (NHS Smoking Cessation Adviser) for inviting patients to participate.

\section{REFERENCES}

1. Killen JD, Fortmann SP, Schatzberg AF, Hayward C, Sussman L, Rothman M, et al. Nicotine patch and paroxetine for smoking cessation. Journal of Consulting and Clinical Pyschology. 2000;68(5):883-9.

2. NHS. Statistics on smoking: England 2018 London: Department of Health and Social Care; 2018.

3. Callum C, Boyle S, Sandford A. Estimating the cost of smoking to the NHS in England and the impact of declining prevalence. Health Economics Policy \& Law. 2011;6(4):489-508. 4. Cahill K, Stevens S, Perera R, Lancaster T. Pharmacological interventions for smoking cessation: an overview and network meta-analysis Cochrane Database of Systematic Reviews. 2013(5):1-52.

5. NICE. Stop smoking interventions and services NICE guideline [NG92]. London: National Institute for Health and Care Excellance 2018. p. 1-33.

6. Stead LF, Lancaster T. Combined pharmacotherapy and behavioural interventions for smoking cessation. Cochrane Database of Systematic Reviews. 2016:1-20. 
572 7. Lacobucci G. Number of people using NHS stop smoking services continues to fall. $573 \quad$ BMJ. 2017;358(j3936):1.

574 8. Hajek P, Phillips-Waller A, Przulj D, Pesola F, et al. A Randomized Trial of E575 Cigarettes versus Nicotine-Replacement Therapy. New England Journal of Medicine. $576 \quad 2019 ; 380:: 629-37$.

577 9. Tamimi N. Knowledge, attitudes and beliefs towards e-cigarettes among e-cigarette 578 users and stop smoking advisors in South East England: a qualitative study. Prim Health Care 579 Res Dev. :1-8. . Primary Health Care Research Development. 2017;19(2 ):189-96.

580 10. Dobbie F, Hiscock R, Leonardi-Bee J, et al. Evaluating long-term outcomes of NHS 581 Stop Smoking Services (ELONS): a prospective cohort study. Health Technology Assessment. $582 \quad 2015 ; 19: 1-156$.

583 11. Osterberg L, Blaschke T. Adherence to medication. New England Journal of Medicine. 2005;353(5):487-97.

585 12. WHO. Adherence to long-term therapies: Evidence for action. Geneva, Switzerland: 586 World Health Organisation 20032003.

587 13. Hazell B, Robson R. Pharmaceutical waste reduction in the NHS. . London: NHS 588 England; 2015.

589 14. Sanders CJ, Lindenmeyer A, Marriott J. A meta-ethnography of adult smokers' 590 exploring the meanings of tobacco dependency medications adherence behaviours during 591 smoking cessation. Journal of Advanced Nursing. 2019;75:3286-98.

592 15. Vrijens B, De Geest S, Hughes DA, Przemyslaw K, Demonceau J, Ruppar T, et al. A 593 new taxonomy for describing and defining adherence to medications. . British Journal of 594 Clinical Pharmacology. 2012;73(5):691-705. 
595

596

597

598

599

600

601

602

603

604

605

606

607

608

609

610

611

612

613

614

615

616

617

618

16. Pacek LR, McClernon FJ, Bosworth HB. Adherence to Pharmacological Smoking Cessation Interventions: A Literature Review and Synthesis of Correlates and Barriers. Nicotine \& Tobacco Research. 2018;20(10):1163-72.

17. Horne R, Chapman SCE, Parham R, Freemantle N, Forbes A, Cooper V. Understanding Patients' Adherence-Related Beliefs about Medicines Prescribed for Long-Term Conditions: A Meta-Analytic Review of the Necessity-Concerns Framework. Plos One. 2013;8(12):1-24.

18. Hollands GJ, McDermott MS, Lindson-Hawley N, Vogt F, Farley A, Averyard P. Interventions to increase adherence to medications for tobacco dependence (Review). Cochrane Database of Systematic Reviews. 2015(2):1-51.

19. Hiscock R, Judge K, Bauld L. Social inequalities in quitting smoking: what factors mediate the relationship between socioeconomic position and smoking cessation? Journal of Public Health. 2011;33:39-47.

20. Paul CL, Ross S, Bryant J, Hill W, Bonevski B, Keevy N. The social context of smoking: A qualitative study comparing smokers of high versus low socioeconomic position. BMC Public Health. 2010;10:2-7.

21. McQuaid EL, Landier W. Cultural Issues in Medication Adherence: Disparities and Directions. The Journal of General Internal Medicine. 2018;33(2):200-6.

22. Michie S, Johnston M, Abraham C, et al. Making psychological theory useful for implementing evidence based practice: a consensus approach. Qualitative and Safety in Health Care. $2005 ; 14: 26-33$.

23. Michie S, Hyder N, Walia A, West R. Development of a taxonomy of behaviour change techniques used in individual behavioural support for smoking cessation. Addictive Behaviors. 2011;36:315-9.

24. West R. Theory of Addiction. Oxford: Blackwell Publishing; 20062006. 
25. Nieuwlaat R, Wilczynski N, Navarro T, Hobson N, Jeffery R, Keepanasseril A, et al. Interventions for enhancing medication adherence (Review) Cochrane Database of Systematic Reviews. 2014;11:1-732.

26. Haynes RB, Ackloo E, Sahota N, McDonald HP, Yao X. Interventions for enhancing medication adherence. Cochrane Database of Systematic Reviews. 2008(2):1-52.

27. Kuntz JL, Safford MM, Singh JA, et al Patient-centered interventions to improve medication management and adherence: a qualitative review of research findings. Patient Education and Counselling. 2014;97(3):310-26.

28. NICE. Medicines adherence: involving patients in decisions about presecibed nedicines and supporting adherence. CG76. London.: National Institue for Health and Clinical Excellence. ; 2009. p. 1-31.

29. Silverman D. Qualitative Research Issues of Theory, Method and Practice. 3rd Edition ed. London: SAGE Publications; 20112011.

30. Smith JS. Qualitative Psychology A Practical Guide to Research Methods. 2nd Edition ed. London: SAGE Publications; 20082008.

31. NCSCT. Standard Treatment Programme. A guide to behavioural support for smoking cessation. Dorcehster, UK.: NCSCT; 2014. p. 1-60.

32. Heatherton TFK, L.T., Fagerström KO. The Fagerström Test for Nicotine Dependence: a revision of the Fagerström Tolerance Questionnaire. British Journal of Addiction. 1991;86:1119-27.

33. Braun V, Clarke V. Succesful Qualitative Research. A Practical Guide for Beginners. London: SAGE; 2013.

34. Lincoln YS, Guba EG. Naturalistic Inquiry. London: Sage Publications Inc; 1985. 
642 35. Tong A, Sainsbury P, Craig J. Consolidated criteria for reporting qualitative research 643 (COREQ): a 32-item checklist for interviews and focus groups. . International Journal of 644 Qualitative Health Care. 2007;19(6):349-57.

645 36. Michie S, West R. Behaviour change theory and evidence: a presentation to 646 Government. health Psychology Review. 2013;7( 1):1-22.

647 37. West R, Brown J. Theory of addiction. 2nd ed. UK: Wiley Blackwell; 2013.

$648 \quad 38$ PHE. Stoptober 2017 campaign evaluation PHE publications; 2018.

649 39. Edwards SA, Bondy SJ, Callaghan RC, et al. Prevalence of unassisted quit attempts in 650 population-based studies: a systematic review of the literature. Additive Behaviours. $651 \quad 2014 ; 39: 512-19$.

652 40. Morphett K, Partridge B, Gartner C, Carter A, Hall W. Why Don't Smokers Want Help 653 to Quit? A Qualitative Study of Smokers' Attitudes towards Assisted vs. Unassisted Quitting. . International Journal of Environmental Research and Public Health. 2015;12:6591-607.

655 41. Smith AL, Carter SM, Dunlop SM, Freeman B, Chapman S. The Views and 656 Experiences of Smokers Who Quit Smoking Unassisted. A Systematic Review of the 657 Qualitative Evidence. . PLOS ONE. 2015;10(5):1-18.

658 42. Balmford J, Borland R, Hammond D, Cummings M. Adherence to and reasons for 659 premature discontinuation from stop-smoking medication: Data from the ITC four-county 660 survey. Nicotine and Tobacco Research. 2011;13(5):94-102.

661 43. Shiffman S. Patterns of over-the-counter nicotine gum use: persitent use and concurrent 662 smoking. Addiction. 2003;98:1747-53.

663 44. Fagerstrom KO, Hughes JR. Nicotine concentrations with concurrent use of cigarettes 664 and nicotine replacement: A review. Nicotine and Tobacco Research. 2002:S73-S9. 
45. Ginsberg JP, Klesges RC, Johnson KC, Eck LH, Meyers AW, Winders SA. The relationship bewteen a history of depression and adherence to a multicomponent smokingcessation program. Addictive Behaviours. 1997;22:783-7.

46. Alterman AI, Gariti P, Cook TG, Canaan A. Nicodermal patch adherence and its correlates. Drug and Alcohol Dependence. 1999;53(2):159-65.

47. Gourlay SG, al. e. Prospective study of factors predicting outcome of transdermal nicotine treatment in smoking cessation. British Medical Journal. 1994;309:842-6.

48. Hartmann-Boyce J, McRobbie H, Bullen C, Begh R, Stead LF, P. H. Electronic cigarettes for smoking cessation. Cochrane Database of Systematic Reviews. Cochrane Database of Systematic Reviews. 2016(9).

49. McNeill A, Brose LS, Calder R, Bauld L, Robson D. Evidence review of e-cigarettes and heated tobacco products. London: Public Health England; 2018

50. King BA, Patel R, H. NK, Dube SR. Trends in awareness and use of electronic cigarettes among us adults, 2010-2013. Nicotine and Tobacco Research. 2014;17(2):219-27. 
Figures

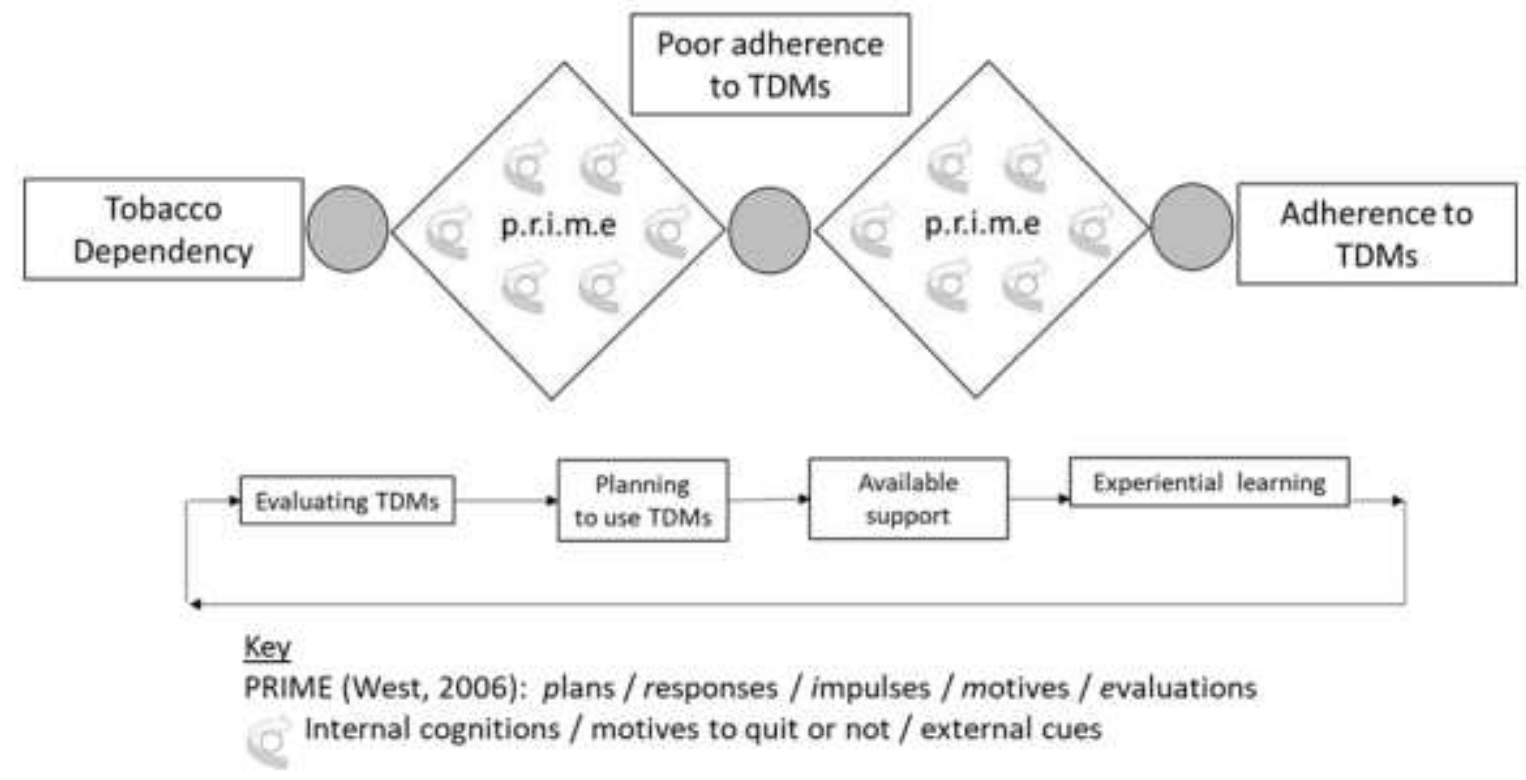

Figure 1

A model of smokers' tobacco dependency medication adherence 